\title{
Carnets
}

Revue électronique d'études françaises de l'APEF

Première Série - 3| 2011

$\mathrm{L}^{\prime}(\mathrm{In})$ vraisemblable

\section{À l'écoute d'Apollinaire: pour revenir au souci de vérité, de vraisemblance qui domine toutes les recherches [...] de l'esprit nouveau}

\section{Alicia Piquer Desvaux}

\section{OpenEdition}

\section{Journals}

\section{Édition électronique}

URL : http://journals.openedition.org/carnets/6017

DOI : $10.4000 /$ carnets. 6017

ISSN : 1646-7698

Éditeur

APEF

Édition imprimée

Date de publication : 2 janvier 2011

Pagination : 115-130

Référence électronique

Alicia Piquer Desvaux, «À l'écoute d'Apollinaire: pour revenir au souci de vérité, de vraisemblance qui domine toutes les recherches [...] de l'esprit nouveau », Carnets [En ligne], Première Série - 3 | 2011, mis en ligne le 18 juin 2018, consulté le 20 avril 2019. URL : http://journals.openedition.org/carnets/6017 ; DOI : 10.4000/carnets.6017

\section{(c) (i) (8)}

Carnets est mis à disposition selon les termes de la licence Creative Commons - Atribution - Pas d'utilisation commerciale 4.0 International. 


\title{
À L'ÉCOUTE D'APOLLINAIRE: \\ Pour revenir au souci de vérité, de vraisemblance qui domine toutes les recherches [...] de l'esprit nouveau
}

Alicia PIQUer Desvaux

Universitat de Barcelona

apiquer@ub.edu

\begin{abstract}
Résumé
On connaît les avatars de la notion de vraisemblance le long de la postmodernité, en France particulièrement. Elle demeure l'apanage des théoriciens qui insistent sur l'(in)vraisemblable. Dénonciation de la fiction, ère du soupçon, jeux d'intertextualité, déconstruction, dissémination, l'arbitraire de la littérature soumise à la littéralité et à l'appréciation du lecteur. Devenu explorateur d'un espace "surréel", Apollinaire cherche, dans ses contes et récits, à dépasser les limites de toute vraisemblance, à découvrir au moyen de l'humour et de l'imagination débridée, des nouvelles approches à une réalité "aux significations multiples".
\end{abstract}

\begin{abstract}
If, during centuries, France has postulated verisimilitude in all literary endeavours as a general rule for lucidity and good taste, throughout postmodernity it has been France's philosophers, semioticians, literary theorists and writers who have questioned this. All fiction is under suspicion; intertextual relations are spoken of, authorship is renounced, emphasis is placed on the arbitrariness of literature that is subjected to literality, etc. Through humour and boundless imagination the short stories of Apollinaire show us a world that goes beyond the limits of the true-to-life; that is, they provide a new approach to a reality "with multiple possible meanings".
\end{abstract}

Mots-clés: Vraisemblance, surréalité, humour, contes, Apollinaire

Keywords: Verisimilitude, humour, postmodernity, short stories, Apollinaire 
Dans l'Antiquité, la vraisemblance avait été définie dans la rhétorique judiciaire (Platon: Gorgias; Corax, disciple d'Empédocle) comme un "art de persuasion", et dans la Poétique d'Aristote, comme une mesure de crédibilité: le rôle du poète serait de dire non pas ce qui a lieu réellement, mais ce qui pourrait avoir lieu. Ainsi expliquée, elle s'est constituée la pierre de touche des poétiques littéraires le long de l'histoire, et a continué à être définie de façon à ne pas exclure les ambiguïtés: la suite des poétiques du $X V I^{e}$ et $d u X V I I^{e}$ siècle (Chapelain, Balzac, Mlle de Scudéry, La Mesnardière, Vossius, Rapin, Corneille, l'abbé d'Aubignac...), ainsi que les différentes œuvres de l'époque, souvent contradictoires, le montrent bien. La modernité romantique force ces présupposés (introduit le grotesque et le sublime), tandis que l'esthétique réaliste prétend à la reproduction "vraie" du monde. Cette illusion mimétique, bientôt déjouée par les écrivains, aboutit au contraire (la mise en œuvre des problèmes de perception et de connaissance). Flaubert, Maupassant, Jarry, les poètes maudits jouaient au moyen de l'ironie, de l'humour, du fantastique, à rendre évident le trompe-l'œil. D'autres, comme Mallarmé, "laissaient l'initiative aux mots". Apollinaire travaillait "l'esprit nouveau"; les surréalistes, en pratiquant "l'écriture automatique" ou en acceptant l'expérience d'une écriture à plusieurs ont contribué à désacraliser la figure de l'auteur; mais c'est bien Borges qui deviendra le plus radical, déjà en plein $\mathrm{XX}^{\mathrm{e}}$ siècle (pensons aux nouvelles qui composent son recueil de Ficciones).

On connaît les avatars de la notion de vraisemblance le long de la postmodernité prônée par Lyotard, en France particulièrement. Elle demeure l'apanage des théoriciens qui insistent sur l'(in)vraisemblable. Dénonciation de la fiction, ère du soupçon, jeux d'intertextualité (Barthes 1980: 1015), déconstruction, dissémination. De Foucault (Les mots et les choses, 1966) à Barthes (le texte "étoilé ou brisé" et "la pluralité de lectures" de "Sarrazine" dans S/Z, 1970), Deleuze-Guattari (Rhizome, 1976), Derrida (De la grammatologie, 1967; La dissémination, 1972), Genette ("Vraisemblance ou motivation" Figures II, 1969; Palimpsestes, 1982), ou Baudrillard (Simulacre et simulation, 1981) tous insistent sur les rapports d'intertextualité, ou sur l'arbitraire de la littérature soumise à la littéralité et à l'appréciation du lecteur et, de surcroît, envisagent "la mort de l'auteur" (Barthes, 1968: 491-495).

Comment le poète Apollinaire avance-t-il ce débat? Apollinaire cherche, dès ses premières expériences littéraires, à dépasser les limites de toute vraisemblance, à découvrir de nouvelles approches à une réalité "aux significations multiples". Cet idéal de modernité est pourtant conséquence moins d'une rupture (façon rimbaldienne) que d'une assimilation et réinterprétation de la tradition pendant ses années de formation littéraire.

Apollinaire, habitué à écrire de petits récits dans les revues de l'époque, réunit et complète ses premiers contes et nouvelles dans des recueils (L'Enchanteur pourrissant (1909), Hérésiarque et CIA (1910), Le Poète Assassiné (1916) qui auront un certain succès 
de critique et vont augmenter sa popularité auprès des cénacles littéraires d'orientation diverse (il est salué comme grand conteur aussi bien par André Billy que par Breton). Moins connus de nos jours puisque la renommée du grand poète d'Alcools et de Calligrammes attire toute l'attention, le travail d'édition des CEuvres Complètes aux éditions de la Pléiade élaboré brillamment par Michel Décaudin (Apollinaire 1993) et les études de Jean Burgos principalement (Burgos 1972), ont contribué à redonner à ces récits l'importance qu'ils ont dans le procès de gestation de l'œuvre entière, ainsi qu'ils montrent la cohérence du discours de l'auteur (dans le sens de la "motivation" dont parle Genette).

\section{L'Enchanteur pourrissant}

L'Enchanteur pourrissant répond à son engouement pour les mythes et les légendes du Moyen âge, les romans de la Table Ronde et la figure de Merlin. II n'est pas le seul: de nombreux ouvrages romantiques sont revenus sur ces personnages merveilleux et courtois. Il y a eu le labeur des érudits qu'Apollinaire connaissait car il aimait fréquenter les bibliothèques dès son arrivée à Paris en 1899. Là il lisait aussi bien les œuvres anciennes rééditées (Lancelot en prose; Merlin Huth; Histoire de Raymondin et de Mélusine, Orlando furioso, Les Romans en vers de la Table Ronde - publiés par Gaston Paris en 1888), que les œuvres des philologues ou des historiens presque contemporains: Myrdhinn ou l'Enchanteur Merlin de Théodore Hersart de la Villemarqué (1882); Le Génie des religions (particulièrement l' “Évangile de l'Enfer") et Merlin, l'Enchanteur d'Edgard Quinet (1842 et 1860) parmi d'autres ouvrages.

Les sources littéraires romantiques et symbolistes avaient redéfini le sens mythique des traditions celtiques, bretonnes et germaniques, leur accordant cette aura d'idéalisme et de mystère de plus en plus éloignée de l'interprétation traditionnelle et chrétienne, plongeant dans les sources philosophiques de Nietzsche, de Schopenhauer, et dans le prestige de "l'art total" wagnérien. Pourtant, contemporain de Wagner, un autre allemand, Friedrich Max Müller, donne une signification totalement différente des mythes. Mallarmé connaît les théories de Müller et de son disciple anglais, G.W. Cox, qu'il traduit dans Les Dieux antiques. II insiste dans ses Divagations: les mythes ne sont que des mots et procèdent d'une dérive inconsciente du langage. À la mythologie wagnérienne (mythologie héroïque, historique, nationale, qui maintient l'illusion du divin), Mallarmé oppose la création d'une mythologie démystifiée, suggérée par la fascination des mots, source de la poésie moderne. D'où la réplique de Mallarmé à propos de Salomé à son ami Lefébure au sujet de la documentation historique que celui-ci s'apprête à lui fournir: "je tiens à en faire un être purement rêvé et 
absolument indépendant de l'histoire"1, car ce n'est plus le réel, historique ou légendaire, qu'il s'agirait de rendre par les mots, mais précisément un mot, un nom, Hérodiade, dont le poème entier ne sera que le déploiement imaginaire. Si Merlin hante l'imaginaire romantique, c'est bien cette danseuse orientale, Salomé, qui va dominer l'imaginaire fin-de-siècle.

En composant L'Enchanteur pourrissant, Apollinaire prétend suivre l'exemple de Mallarmé et réécrire un texte désormais nouveau sur Merlin. D'autre part, la femme fatale hante la totalité de l'œuvre d'Apollinaire, elle aussi réinventée poétiquement.

Outre sa grande érudition, d'autres aspects motivent le goût d'Apollinaire envers la légende de Merlin. Lors d'un séjour dans la petite ville belge de Stavelot, avec son frère, l'été 1899, il s'enivre du charme des forêts. La forêt des Ardennes évoque pour lui le décor de la forêt bretonne de Brocéliande ${ }^{2}$. Tout comme l'histoire de l'"enfant sans père" s'assimile à sa propre histoire personnelle. Le nouveau récit sur le mage, le druide Merlin, reprend pour la transformer l'allégorie de l'Anti Christ moyenâgeux. II devient un "texte hybride" qui pourrait correspondre à ce que bien plus tard Jean-Yves Tadié nommera "récit poétique"3, forme d'écriture qui conserve la fiction du roman, mais dont les procédés narratifs renvoient au poème. De façon à créer une tension entre la fonction de représentation référentielle et la structure particulière du message dominée par les images, les symboles ou la musicalité. Tension ou conflit que l'on peut ressentir avec ces tableaux "où la représentation lutte avec l'abstraction" (Tadié 1978: 12). Récit poétique donc, semblable à "une fantaisie de Noël funéraire", avec trois rois mages qui disparaissent "par enchantement" (Apollinaire 1977: 29). Le caractère illusoire, donc littéraire, est bien mis en relief, ce qui accorde au poète la grande liberté de combiner les éléments de la légende (l'incantation et la danse de Viviane, Merlin amoureux de Viviane, la Dame du $\mathrm{Lac}^{4}$, le magicien qui parle aux animaux, l'enchanteur enchanté), avec les inventions de son propre cru: nous trouvons un "Myrdhinn" (le nom à la mode bretonne, que Hersart de la Villemarqué avait récupéré) vu de l'intérieur, avec ses vifs sentiments et ses contradictions, condamné à vivre sans vivre, enfoui par Viviane dans le tombeau. Plus homme que diable, il va venir rejoindre d'autres figures mythiques et littéraires (bibliques, gréco-latines - Orphée, Hélène de Troie -, orientales, et propres du cycle d'Arthur, comme celle du monstre Chapalu; Angélica, l'héroïne de l'Orlando Furioso de l'Ariosto) au sort semblable, en parfait syncrétisme, avec lesquels il établira un dialogue qui

\footnotetext{
${ }^{1}$ Lettre de Mallarmé à son ami Lefébure, février 1865.

2 L'histoire moderne du tombeau de Merlin commence, en France, en 1820. Un érudit, J.C.D. Poignard, écrit, à partir de certains témoignages et de la tradition orale, que Merlin aurait été enterré dans la forêt de Paimpont (aujourd'hui commune de Saint-Malon-sur Mel, au département d'llle-et-Vilaine, Morbihan, Bretagne), où se situerait la forêt légendaire de Brocéliande. En 1889, Félix Bellamy, écrivain spécialisé dans la légende arthurienne, y "trouva" le tombeau, transformé aujourd'hui en lieu touristique.

3 "texte qui se présente comme un lieu d'échange entre récit (considéré en tant qu'épure du roman) et poème" (Tadié 1978: 6)

${ }^{4}$ La Dame du Lac connut un regain de faveur à partir du long poème The Lady of the Lake, que Walter Scott composa en 1810. II inspira l'opéra La Donna del Lago de Rossini (1819), et les Ellens Gesang I, II, III, de Schubert.
} 
tourne en rond sur les énigmes de la vie, de la mort et de l'amour, ou de l'impossibilité de l'amour. Chaque réplique aura, quoiqu'en prose, la solennité rythmique de l'incantation, et les voix, s'organisant autour de celle de Merlin, sembleraient un chœur dramatique...si ce n'était que le lecteur se laisse aller à la dérive proposée par les mots, tantôt aux associations pittoresques qu'érudites, souvent inattendues. Le chant de toutes ces figures symboliques, venues des lieux et des temps différents, évoque bien une pratique du simultanéisme qui va désormais caractériser l'œuvre entière d'Apollinaire.

L'honneur du simultanéisme pictural (par la couleur) reviendra à Delaunay ${ }^{5}$, mais le simultanéisme littéraire aurait des origines plurielles, malgré les protestations d'Apollinaire, qui se considère le premier à l'employer. René Ghil avait fait des recherches "instrumentalistes" (l'émission simultanée de plusieurs voix indépendantes qui visait à restituer la complexité "polyphonique" du réel), qu'Henri Martin Barzun et les écrivains autour de la revue Poème et Drame approfondirent, dans la ligne des futuristes ${ }^{6}$. Apollinaire travaillait spécialement et dès les premières versions de L'Enchanteur pourrissant à saisir la primauté de l'instant et l'éternité du temps ${ }^{7}$. Bien que ce sont les poèmes d'Alcools (1913) "Zone" ou "Vendémiaire", par exemple - qui vont le mieux associer à l'expression de l'ensemble du temps, la réflexion sur l'espace multiple, visible aussi dans l'éclatement de la mise en page tellement caractéristique.

Quoiqu'il en soit, L'Enchanteur pourrissant, conçu dès 1899 allait connaître plusieurs versions: celle de 1901; celle de 1903, à son retour d'Allemagne; sa première publication dans la revue fondée par Apollinaire en 1904, Le Festin d'Ésope, qui présente l'abandon de quelques chapitres, repris par la suite, convenablement modifiés, dans Le poète assassiné (le thème de Merlin nourrit aussi des poèmes d'Alcools). Finalement, en 1909, le texte est publié illustré avec 32 bois gravés d'André Derain ${ }^{8}$. La version définitive (Apollinaire 1993: 580) comprend une introduction qui suit de près le Lancelot en prose du $\mathrm{XVI}^{\mathrm{e}}$ siècle et un dernier chapitre tout à fait invention du poète, Onirocritique, qui avait vu sa publication le 15 janvier 1908 dans La Phalange.

\footnotetext{
${ }^{5}$ Robert et Sonia Delaunay rêvaient d'une exposition universelle simultanéiste, qui devait être commentée par Cendrars. Finalement elle eut lieu (de façon plus modeste) à la galerie Dalmau de Barcelone, en 1914.

${ }^{6} \mathrm{La}$ "vision multiple et totale de I'Individuel, du Collectif, de l'Humain et de l'Universel' débouche sur le chant polyphonique d'une nouvelle poésie dont le programme (L'Ére du drame, 1912) fut élaboré par Barzun: son "dramatisme" (devenu simultanéisme par la suite) se veut la synthèse de diverses tendances de l'avant-garde littéraire, telles qu'elles étaient définies par les poètes de l'Abbaye, sous le nom d'instrumentalisme, d'unanimisme ou de futurisme. Barzun appartenait aussi au groupe des poètes typographes œuvrant à la représentation de la complexité accrue du monde moderne.

7 “'L'image temps, l'image mouvement" (Deleuze 1983)

${ }^{8}$ L'éditeur, D. Kahnweiler, avait déjà demandé à Apollinaire de préfacer le catalogue de sa première exposition, consacrée à Braque. II prétendait éditer des œuvres de jeunes écrivains illustrées par des peintres nouveaux. Apollinaire avait une connaissance profonde du travail des peintres cubistes, ainsi que de solides rapports d'amitié avec le groupe du Bateau-Lavoir.
} 
Ainsi composé, par "assemblage" (Décaudin), le texte offre tantôt la citation, tantôt la réécriture ou la variante, et, finalement, la création moderne qui dépasse les modèles traditionnels.

Le court récit d'Onirocritique (dont le titre nous introduit directement dans le monde des rêves et de son interprétation) nous présente ce double moderne de Merlin, qu'est le poète, capable de voyance: "Les charbons du ciel étaient si proches que je craignais leur ardeur. Ils étaient sur le point de me brûler. Mais j'avais la conscience des éternités différentes de l'homme et de la femme" (Apollinaire 1993: 73).

La vision d'un monde nouveau, aux animaux fabuleux, au Temps renversé ("le faux hiver") semble convenir au narrateur qui a l'aspect étrange d'un géant à grand appétit ("J'avalai des troupeaux basanés"). Image insolite qui prendra avec le développement de l'ensemble de l'œuvre toute sa signification (le Chroniamantal du Poète assassiné; le géant rabelaisien de "Vendémiaire", capable de "boire l'univers"). Épris de curiosité il rentrera dans la ville d'Orkenise, suivant la chanson qu'il entend chanter, il apportera son cœur pour se marier. Mais là il découvre une terre toute fertile où le principe de la Genèse s'accomplit: tout pousse, grandit, grossit, plantes et animaux. Les hommes aussi et lui-même:

Vers le soir, les arbres s'envolèrent, les singes devinrent immobiles et je me vis au centuple. La troupe que j'étais s'assit au bord de la mer. De grands vaisseaux d'or passaient à l'horizon. Et quand la nuit fut complète, cent flammes vinrent à ma rencontre. Je procréai cent enfants mâles dont les nourrices furent la lune et la colline (Apollinaire 1993: 74)

Mais de l' "illumination" notre poète vire rapidement au cauchemar: il brandit "un fleuve comme une épée" pour se désaltérer et les hommes tuent toutes ses vies, sauf une. II parvient à se sauver, continue à parcourir ce monde luxurieux, qui offre ses beaux fruits. II terrasse un autre homme, des serpents sortent de la bouche "et leur langue s'appelait SaintFabeau" ${ }^{9}$. Finalement il demeure seul dans un monde abandonné.

Dans Onirocritique la valeur sexuelle de l'épée s'associe à l'image du serpent et "est complétée par celle de la parole: Sainte Fabeau est le nom de la langue des serpents, mais le mot est aussi composé sur un radical fab, qu'on retrouve dans fabula, fable", nous dit Décaudin (Apollinaire 1993: 1107). Dans "Les Collines" (Calligrammes), l'image récurrente de l'épée est bel et bien associée au rôle du poète voyeur-prophète-créateur de nouveaux mondes:

${ }^{9}$ L'hermétisme est moindre si nous suivons le parcours dans l'œuvre d'Apollinaire de certains motifs réitérés: nous retrouvons dans "La chanson du mal-aimé" (Alcools) le thème biblique de sept épées qui traversent le cœur de Marie, dont la cinquième épée s'appelle Saint-Fabeau. 


\author{
$[\ldots]$ \\ Certains hommes sont des collines \\ Qui s'élèvent d'entre les hommes \\ Et voient au loin tout l'avenir \\ Mieux que s'il était le présent \\ Plus net que s'il était passé \\ [...] \\ L'esclave tient une épée nue \\ Semblable aux sources et aux fleuves \\ Et chaque fois qu'elle s'abaisse \\ Un univers est éventré \\ Dont il sort des mondes nouveaux
}

Apollinaire travaille donc à une poétique rigoureuse où les réseaux des significations se constituent le long de l'œuvre par de liens subtils autotextuels et intertextuels, car de la même façon qu'il réécrit la légende de Merlin dans L'Enchanteur pourrissant, il fait appel à la tradition talmudique dans Onirocritique, pour bien souligner l'importance du projet divin (Dieu dit que la terre produise des êtres animés selon leurs espèces, l'homme donc souhaite réaliser ce projet).

Onirocritique permet de renverser la tragédie de Merlin (toujours en échec devant la femme fatale, toujours criant des questions sans réponse, lui qui aurait dû tout savoir): le dernier chapitre présente la toute-puissance du poète-créateur seul face au monde et au temps". Ce triomphe surnaturel du Poète face au Destin devient un thème fondamental chez Apollinaire (nous pensons à "Vendémiaire" qui termine Alcools et au "Musicien de SaintMerry" dans Calligrammes).

\title{
L'Hérésiarque et Cie
}

Devenu journaliste chez L'Intransigeant, en 1910, Apollinaire combine son travail avec une grande production littéraire, qui va cristalliser dans un recueil qu'il propose à l'éditeur Stock avec le titre de Phantasmes, bientôt modifié. Resté en troisième place du prix Goncourt, sa désillusion est moindre en voyant le bon accueil de la critique. Certains interprétaient le livre à la lumière de possibles influences de Baudelaire, Nerval ou Hoffmann, qu'Apollinaire refuse catégoriquement. Pourtant cela semblerait bien possible vu la façon de travailler ses thèmes et son écriture, qui abondent en références littéraires. Le ton général du livre unifie la diversité des récits, mais s'agit-il de contes ou de nouvelles? Genre ce dernier qui a une grande popularité à l'époque. Le choix du genre a son 
importance, car il véhicule la fusion du réel et de l'imaginaire, essentielle dans l'univers d'Apollinaire.

À différence du conte qui garderait ses origines externes au narrateur et son caractère plus féerique, nous pensons que nous pouvons ici parler carrément de nouvelles: la voix du personnage-narrateur et son point de vue menant le récit. C'est au fil de ses rencontres lors de ses voyages ou au fil de ses lectures que l'intrigue surgit. Apollinaire mêle à la narration des éléments de légende populaire et des anecdotes tirées de la vie quotidienne, les fusionne si bien que l'imaginaire et la réalité semblent naturellement aller de pair.

La plupart de ces nouvelles situent leurs intrigues dans le monde réel, quoique cette réalité évoquée présente l'irruption de l'insolite. Cette apparition inespérée est rendue encore moins inexplicable à cause de la brièveté de l'ensemble raconté, de l'ellipse des détails ou de l'accélération dramatique des scènes. Incompréhensible et plein d'ambiguïté, l'insolite inquiète. Pris au dépourvu personnages, narrateur, narrataire sentent le danger, devinent les conséquences funestes. Quant on considère un fantastique traditionnel, on établit bien l'écart avec le féerique, univers merveilleux qui s'ajoute au monde réel sans en détruire la cohérence: le conte de fées, les légendes se déroulent dans un temps éloigné, dans un monde où l'enchantement va de soi et où la magie est d'usage. Apollinaire avait déjà dérangé l'harmonie du féerique dans ce "récit poétique" que nous considérons L'Enchanteur pourrissant. Avec L'Hérésiarque et Cie (Apollinaire 1993: 81-225) il plonge dans l'univers du fantastique. Fantastique qui, après avoir introduit un élément étranger dans l'intrigue, brise toute cohérence, termine de façon sinistre, même si l'ordre des choses reprend par la suite: la perception que l'on peut en avoir reste profondément troublée.

Dans "Le passant de Prague", Apollinaire, venant de Berlin par Dresde, a fait un séjour relativement court, qui lui a permis de bien connaître les endroits les plus pittoresques de la ville, notamment son quartier juif. C'était en mars 1902. Puis il part pour Vienne. En tout cas le conte est publié le $1^{\text {er }}$ juin dans La Revue Blanche avant d'être intégré au recueil de L'Hérésiarque et Cie: le poète débarque à Prague qu'il veut visiter. Voici qu'il fait la rencontre d'un type "étranger comme vous" qui lui fait voir les coins de la ville, le quartier juif, qui devient rouge la nuit. La tour de l'Hôtel de Ville dont les aiguilles de l'Horloge marchent à rebours, etc. La description des lieux est assez précise, mais la personnalité du guide devient de plus en plus inquiétante: il marche pendant des années et des siècles sans jamais s'arrêter, sans jamais mourir. Son nom est Isaac Laquedem (le même personnage qui dialogue avec l'enchanteur pourrissant et d'autres illustres prétendus immortels: Empédocle, Énoch, Élie, Simon le Magicien, les trois faux rois mages), bien qu'on le connaisse partout sous le nom du Juif errant, et sous bien d'autres appellations qui nous renseignent sur l'étendue populaire de la légende. Devant l'ahurissement du poète, il avoue 
qu'il passera sous silence des éléments de son identité "sinon que Jésus m'ordonna de marcher jusqu'à son retour". II ignore les œuvres qu'il a inspirées, mais en connaît les auteurs qu'il débite devant son touriste. La liste est très longue (plus de 31 écrivains cités): de Goethe et Schlegel, passant par Andersen, jusqu'à Edgard Quinet, Eugène Sue, Gaston Paris, Jean Richepin... Laquedem continue: "Il est juste d'ajouter que tous ces auteurs se sont aidés du petit livre de colportage qui, paru à Leyde en 1602, fût aussitôt traduit en latin, français et allemand, et fut rajeuni et augmenté par Simrock dans ses livres populaires allemands" (Apollinaire 1993: 87).

Le texte continue de combiner le récit du tour de la ville avec les éléments de cette légende qui existe depuis la nuit des temps en Europe. Ici, maintenant, partout ailleurs et toujours. La fin du récit est assez libre mais toujours inquiétante, ainsi que la morale qui s'en dégage des lèvres même de Laquedem drôle et bizarre en même temps: "Des remords? Pourquoi? Gardez la paix de l'âme et soyez méchant [...] Le Christ je l'ai bafoué. II m'a fait surhumain".

"Le Sacrilège", "Le Juif latin", "L'Hérésiarque" et "L'infaillibilité" tournent autour de questions de dogme catholique, de foi, des relations entre le judaïsme et la latinité, de discipline ecclésiastique; des divergences sur l'interprétation des livres saints, des hérésies. Les récits peuvent nous sembler fantaisistes, il n'empêche qu'ils dégagent les faiblesses et la perversité de l'homme, comme c'est le cas du "Juif latin", dont le dénouement paradoxal et incongru rappelle le style d'Alphonse Allais: il nous fait sourire, mais sème le doute à propos de l'équité divine. Ou à propos de I'hérésie de Benedetto Orfei (“L'Hérésiarque"): "La vérité est que l'hérésiarque était pareil à tous les hommes, car tous sont à la fois pêcheurs et saints, quand ils ne sont pas criminels et martyrs". En tout cas, ces récits semblent répondre à l'intérêt soulevé dans la presse par les conversations secrètes qui eurent lieu entre Paris et le Vatican après la loi de séparation entre l'Église et l'État (1905), sans oublier le climat de controverse autour de l'affaire Dreyfus.

Apollinaire continue à s'inspirer des légendes populaires européennes mises à jour par les romantiques, mais aussi d'histoires réelles ${ }^{10}$ ou tirées de livres érudits ("Simon le Mage"11, "La danseuse" sur la mort de Salomé). L'attention qu'il prête pourtant aux rêves des protagonistes, ou à leur imagination, découvre bien les intentions de l'auteur, qui cherche à manifester l'importance d'autres états de conscience pour aller plus loin que les arguments de la raison.

\footnotetext{
10 "L'infaillibilité" est axée sur le dogme pontifical proclamé en 1870 par le premier concile du Vatican, qui trouva grand écho dans la presse de l'époque. Dans “Le Giton” Apollinaire évoque le vieux Nice, qu'il connaît bien.

11 A partir du Vocabulaire de l'angélologie de Moïse Schwab (1897), qu'il avait lu, Apollinaire fait une description des anges aidant Simon le Mage (ou Simon de Samarie) à s'envoler, description qu'il considère comme une de ses pages le mieux réussies. Voir la note de Decaudin (Apollinaire 1993: 1127).
} 
L'essor de la nouvelle fantastique est conséquence directe de la croyance dans le surnaturel que le romantisme avait renforcé et du scientisme et positivisme dominant la finde siècle: l'apparition de personnages légendaires au coin d'une rue quelconque, la folie, la magie, les hallucinations, les revenants, les spectres, les morts-vivants, les doubles, autant de situations et d'images qui peuvent bouleverser ou inquiéter précisément parce qu'ils partent d'éléments anodins de la vie quotidienne. Si Baudelaire aimait à y faire étalage de "la méchanceté naturelle de l'homme", Apollinaire dit ceci ("Le juif latin", "Le matelot d'Amsterdam", "Histoire d'une famille vertueuse, d'un hôte et d'un calcul"), mais aborde aussi le thème de l'ambiguïté des contraires ("Le giton", "Que vlo-ve?"), et surtout, s'il utilise parfois le fantastique pour montrer que les superstitions sont un leurre et que la connaissance scientifique montrerait la possibilité de certains phénomènes ("La disparition d'Honoré Subirac" ${ }^{12}$ ), le résultat est absurde ou comique, ou simplement le mystère s'explique à moitié ("La Rose d'Hildesheim ou les Trésors des rois mages")

Pourtant, nous allons retenir "L'Amphion faux messie ou histoires et aventures du baron d'Ormessan", spécialement ce passage où d'Ormessan, afin de tourner un film bien vrai, décide, pour que la scène soit vraisemblable, que les acteurs s'entre-tuent. Ceci nous fait penser à tout un jeu d'interprétation de l'art comme représentation de la "réalité". De façon détournée, Apollinaire serait en train de condamner le "réalisme" comme principe esthétique. Mais il condamnerait également le "symbolisme", esthétique fondée sur l'idéalisme, de la même façon qu'il dénonce et emploie à son gré les légendes, les histoires surnaturelles ou les miracles.

\section{Le poète assassiné}

Le poète assassiné est publié en octobre 1916, mais sa genèse a été complexe. Apollinaire est déjà bien connu dans les cénacles littéraires et artistiques après la publication, en 1913, d'Alcools et de Méditations esthétiques. Les peintres cubistes. Le recueil du Poète assassiné contient, en suivant la technique d'assemblage, des textes composés dans le passé, principalement des récits qu'il a éliminés du recueil définitif de L'Enchanteur pourrissant ou qui n'avaient pas la place dans L'Hérésiarque et Cie, et d'autres absolument nouveaux, puisqu'il avait commencé la rédaction d'un prétendu roman (Le roi lune, sur Louis II de Bavière ${ }^{13}$ ) et un autre roman qui aurait dû s'appeler Le Poète assassiné.

II s'agit d'une suite de chapitres qui expliquent la généalogie, la naissance, la vie et la mort de Croniamantal, le poète admiré et détesté à la fois. Récit d'initiation, quête de l'amour

\footnotetext{
${ }^{12} v$, "La disparition d'Honoré Subirac": il est capable de devenir invisible par l'effet de la panique, à l'instar de tellement d'insectes ou d'autres animaux qu'à la vue du danger peuvent se camoufler.

${ }^{13}$ Par opposition à Louis XIV, le Roi-Soleil.
} 
et de la Poésie, qui peut être lu comme une autobiographie caricaturale (Apollinaire avait aussi entamé un texte sur sa jeunesse qu'il aurait intitulé "Histoire de Nyctor") et une démythification ubuesque du Poète. Exercice d'humour généralisé et d'ironie qui découle des caractéristiques du personnage, spécialement du nom choisi ${ }^{14}$. Le texte regorge de trouvailles ingénieuses de toutes sortes, les unes portant sur des calembours, les autres sur les situations équivoques, dans la ligne cocasse de "Que vlo-ve?".

Comme le fantastique, le rire grotesque et satanique (Baudelaire, 1968: 373) et I'humour plus subtil attirent l'attention des artistes et du public. Les théories prolifèrent sur l'humour autant que sur le fantastique le long du $\mathrm{XIX}^{\mathrm{e}}$ siècle (le débat se poursuit encore). Apollinaire se positionne aussi, à partir de son travail créatif: I'humour n'a pas besoin de conventions, donc de vraisemblance, d'autant plus qu'il est fondé la plupart des fois sur la surprise. Cherchant à parfaire sa propre esthétique, Apollinaire fait une entorse de plus aux notions de vraisemblance, réalisme ou vérité en art, et ouvre la porte à l'imagination débridée.

II s'agit d'abord de nous présenter la "renommée" du Poète: "La gloire de Croniamantal est aujourd'hui universelle. Cent vingt-trois villes dans sept pays sur quatre continents se disputent l'honneur d'avoir vu naître ce héros insigne". L'hyperbole qui se développe le long du récit et au large des différents pays, accumulant les diverses dénominations selon les différents lieux, nous permet de considérer le poète à la fois dans le Temps et au-delà du Temps: être historique et héros mythique à la fois. Nous reconnaissons bien le thème du simultanéisme.

Les parents de Croniamantal sont un musicien wallon et une cycliste "formée de jolis globes" (c'est l'annonce des mamelles de Tirésias), Macarée, qui songe d'abord à l'avortement, connait ensuite un baron français naïf et bon vivant qu'elle décide d'épouser. La naissance a lieu "d'un pet" de son père qui fit rire aux larmes Macarée, avant de mourir. Élevé par son père et un précepteur, le jeune Croniamantal n'a d'autre intérêt que ses études, jusqu'au moment où la nostalgie de l'amour lui serre le cœur. À Paris, il fait la connaissance de "l'oiseau de Benin" (Picasso). II s'éprend de Tristouse (Marie Laurencin) et lui dédie des vers qui la célèbrent et la rendent immortelle. Mais elle le trompe au bout d'une semaine, car les poètes sont trop sérieux. Croniamantal part voyager à travers l'Europe, sur les pas de Tristouse. Plus elle cherche à le tromper, plus la renommée de notre héros est grande. Tristouse incarne la femme fatale. Au milieu du récit, Apollinaire substitue à l'humour gros sel des références à l'art nouveau qu'il prétend écrire. D'où la référence à Picasso et

\footnotetext{
${ }^{14}$ Le nom serait la contraction de Cro-Magnon et Neandertal (Apollinaire 1993: 1160). Nous retrouvons la fusion des éléments historiques et proprement inventés par l'écrivain. La date de 1868 n'est pas si éloignée où l'on trouva à Cro-Magnon, en Dordogne, des restes fossiles d'une population d'Homo sapiens, qui peuplait l'Europe centrale et occidentale au Paléolithique supérieur. Ce fut en 1856, dans le site préhistorique de Neandertal, près de Düsseldorf, que l'on découvrit le premier squelette fossile humain reconnu différent de l'homme actuel, ayant peuplé le Proche-Orient et l'Europe entre 120.000 et 35.000 ans av. J.-C.
} 
l'art africain et l'annonce de Croniamantal de faire du théâtre, après l'échec de son poème pur (Apollinaire 1993: 258). Finalement il meurt aux mains de la foule déchaînée (tel un Orphée moderne), l'œil crevé par le parapluie de Tristouse, qui lui préfère un poète charlatan. L'humour devient noir.

Le deuxième volet correspond au Roi Lune. À nouveau nous accompagnons le narrateur dans son périple: "Le 23 février 1912, je parcourais à pied cette partie du Tyrol". Son errance le conduit à la découverte d'un lieu extraordinaire dans le cœur de la montagne, un scénario spectaculaire où des jeunes gens mangent, boivent, jouent aux cartes, dansent. Bien que choqué par certains meubles et par un banquet cruellement surprenant, notre voyeur continue curieux à regarder. Malgré les indices inquiétants, le récit ne cherche pas à approfondir sur des aspects sataniques ou surnaturels, il s'agit plutôt de développer l'espace du rêve, de s'initier dans une quête de soi et une recherche esthétique nouvelle. Des lieux vont défiler, que nous saisissons comme vraiment autobiographiques (lieux et paysages d'enfance: "Giovanni Moroni"). Les récits se suivent sans progression, mais des lignes de force se dessinent: quelques-unes on les connaît, la prédilection d'Apollinaire pour les personnages mythiques (Arthur, roi passé roi futur) ou mythifiés (Le roi Lune); ou ceux dont l'identité reste floue, espèce de revenants détournant les coordonnées espace-temps; les jeux de magie, et, surtout, les amours tragiques (toutes les pratiques de la tendresse à l'érotisme et au sadisme sont suggérées, tandis que l'amour vrai dégénère toujours), comme pour Croniamantal, l'amour et la mort vont de pair.

Surtout ce dernier recueil propose deux volets complémentaires de la création poétique: l'un diurne, l'autre nocturne, puisque "Le roi lune" introduit de façon presque systématique la dimension du rêve, comme une possibilité de connaissance, de perception différente d'autres réalités purement subjectives liées aux souvenirs, à l'enfance, au cauchemar. Une métaphore réitérative ${ }^{15}$ insiste sur cette déréalisation: l'évocation récurrente du "vide" (le tombeau vide de Croniamantal, façon de fonder la poésie et la gloire du poète sur rien, image qu'il faut comprendre dans le sens de Flaubert, quand il affirmait son souhait "d'écrire un livre sur rien").

La façon d'insister sur la mise en relief du travail textuel à partir de différents registres (lyrique, féerique, humoristique, absurde, fantastique) nous éloigne de toute interprétation "réaliste" ou "vraisemblable". La poétique d'Apollinaire, gorgée de mythes et de légendes, se passait de vraisemblance, au nom de cette modernité qu'il appellera vers la fin de sa vie |'"esprit nouveau".

\section{Des Mamelles de Tirésias à l'Esprit Nouveau}

\footnotetext{
${ }^{15}$ Voir à ce propos le conte Mon cher Ludovic paru en 1917 dans l'Almanach des lettres et des arts (Apollinaire 1993: 497-499).
} 
Le 24 juin 1916 c'est la première des Mamelles de Tirésias (Apollinaire 2001: 865913) jouée au Conservatoire Renée Maubel à Montmartre. Elle obtient l'incompréhension généralisée d'un nombreux public dérouté devant les ingéniosités jouées (des enfants qui naissent des papiers de journal; un espace équivoque, Paris ou le Zanzibar; des rôles sexuels changés).

Le Prologue en vers (Apollinaire 2001: 879-882) fait parler le directeur d'une troupe de théâtre sur la guerre ${ }^{16}$, sur le sujet de l'œuvre (faire des enfants puisque la France en a besoin après la guerre) et la nécessité "d'infuser un esprit nouveau au théâtre": il faut parvenir à utiliser un mélange de tonalités du pathétique au burlesque afin de marier "sans lien apparent comme dans la vie" les éléments divers du spectacle, sans le souci de "photographier ce que l'on appelle une tranche de vie". Une deuxième idée vient compléter celle-ci, c'est la liberté de l'auteur, véritable "dieu-créateur/ Qui dispose à son gré/ Les sons les démarches les masses les couleurs" à l'intérieur de sa pièce.

Devant les critiques qui arrivaient à détourner complètement ses propos, Apollinaire s'explique lors d'une conférence qui, à la manière d'un manifeste, insiste sur les données théoriques parues dans le Prologue de la pièce. II la rajoutera comme Préface au texte un an après la première représentation, en 1918 (Apollinaire 2001: 865-870). II est question, évidemment, de chercher une rénovation du théâtre, mais les propos soutenus vont éclairer l'œuvre entière.

Comment justifier "l'usage raisonnable des invraisemblances"? Comment éviter l'accusation d'absurde? Ou bien pire, celle d'utiliser l'idée de surréalité l'assimilant au symbolisme, lui qui refuse tout idéalisme? Apollinaire récuse un à un les griefs dont les critiques l'accusent. Son "drame surréaliste" essaye:

de revenir à la nature même, mais sans l'imiter à la façon des photographes. Quand l'homme a voulu imiter la marche, il a crée la roue qui ne ressemble pas à une jambe. II a fait ainsi du surréalisme sans le savoir (Apollinaire 2001: 865-6).

La dramaturgie proposée prétend présenter des mondes nouveaux qui élargissent les horizons, au moyen des découvertes les plus surprenantes. II avait même songé à "deux scènes" emboîtées, qui nous font évoquer certaines propositions des plus hardies d'aujourd'hui, de façon à intégrer le public dans l'action jouée et permettre ainsi "le grand

\footnotetext{
${ }^{16}$ Ici, ce sont les coups de canons qui "éteignent les étoiles", "ils ont même assassiné les constellations". Dans Calligrammes, poèmes de la même époque, ces mêmes canons dessinent des feux d'artifice dans le ciel: "Merveille de la guerre", Obus couleur de lune (Apollinaire 2001: 271)
} 
déploiement de l'art moderne". Tout pour nier le naturalisme, le réalisme, le symbolisme ${ }^{17}$, mouvements tous qui sont axés sur la description, la représentation, l'imitation ou la mimésis de la "réalité", simples "natures mortes". II insiste sur ses conceptions artistiques, toujours prônant la liberté totale de l'artiste. On écoute les échos de Nietzsche, quand dans La Naissance de la tragédie et dans Le Crépuscule des Dieux, déclarait la seule existence du "monde apparent" et considérait le monde "vrai" comme un "mensonge".

C'est à partir de ces considérations que nous pouvons lire L'esprit nouveau et les poètes, qui est à la poésie ce que le Prologue était pour le théâtre, afin d'essayer de résoudre certaines contradictions ou relever de simples ambiguïtés autour de la problématique de la vérité de l'art.

Cette nouvelle conférence donnée en novembre 1917 (Apollinaire 1994: 941-954) affirme comment les poètes français de l'esprit nouveau cherchent à "explorer la vérité" dans tous les domaines, principalement dans celui de "l'imagination". Ils vont utiliser toutes les ressources formelles possibles (vers libre, artifices typographiques, synthèse des arts, le cinéma, le phonographe, les journaux, les nouvelles machines) afin de créer la surprise: montrer comment les mots, dans un contexte imprévisible, peuvent prendre des significations inouïes. Bien sûr, il faudrait éviter que cette synthèse hétéroclite d'éléments puisse dégénérer en une confusion que le public n'accepte pas. Mais les poètes de l'esprit nouveau, comme les alchimistes de jadis, doivent risquer et "s'adonner à des recherches, à des notations qui les [mettraient] en butte aux railleries de leurs contemporains, des journalistes et de snobs".

Comme les mathématiciens capables par leur imagination d'inventer des théories (Newton) et des machines adaptées à la modernité, le poète ne doit pas "méconnaître la magnifique exubérance de vie que les hommes par leur activité ajoutent à la nature et qui permet de machiner le monde de la façon la plus incroyable".

Les poètes de l'esprit nouveau sont ceux qui s'adaptent "au temps même où nous vivons":

On peut partir d'un fait quotidien: un mouchoir qui tombe peut être pour le poète le levier avec lequel il soulèvera tout un univers [...] C'est pourquoi le poète d'aujourd'hui ne méprise aucun mouvement de la nature, et son esprit poursuit la découverte aussi bien dans les synthèses les plus vastes, les plus insaisissables: foules, nébuleuses, océans, nations, que dans les faits en apparence les plus simples [...] C'est pourquoi les poètes modernes sont avant tout les poètes de la vérité toujours nouvelle (Apollinaire 1994: 950).

\footnotetext{
${ }^{17}$ Apollinaire considère le Symbolisme attaché à la croyance d'une vérité absolue (une espèce de réalité suprême, donc) caractéristique de l'idéalisme platonicien.
} 
Cette "vérité" que le poète cherche va contre la vérité communément admise, celle du bon sens et de l'expérience, celle de la vraisemblance:

On peut également exprimer une vérité supposée qui cause la surprise, parce qu'on n'avait pas encore osé la présenter. Mais une vérité supposée n'a point contre elle le bon sens, sans quoi elle ne serait plus la vérité, même supposée. C'est ainsi que si j'imagine que, les femmes ne faisant point d'enfants, les hommes pourraient en faire et que je le montre, j'exprime une vérité littéraire qui ne pourra être qualifiée de fable que hors de la littérature, et je détermine la surprise. Mais ma vérité supposée n'est pas plus extraordinaire, ni plus invraisemblable que celles des Grecs [...] Tant que les avions ne peuplaient pas le ciel, la fable d'Icare n'était qu'une vérité supposée. Aujourd'hui, ce n'est plus une fable (Apollinaire 1994: 948).

Apollinaire n'était pas homme à théories ou manifestes, pourtant il définit avec une grande clarté dans ses écrits sur la création artistique (peinture, théâtre, poésie) l'exigence et la finalité d'un art futuriste, adapté au monde nouveau. À ceux qui vont nier l'utilité de l'art dans les sociétés modernes, il leur répond que "les fables s'étant pour la plupart réalisées et au-delà c'est au poète d'en imaginer des nouvelles que les inventeurs puissent à leur tour réaliser".

Nous avons vu comment dans ses nouvelles les plus connues il était déjà en train d'élaborer et de mettre en pratique les principes esthétiques que par la suite il définit dans sa conférence. Des principes qui ont nourri et accordé une grande cohérence à l'ensemble de son œuvre. Grâce à l'imagination et à la rêverie, le poète devient familier "d'une immensité inconnue où flambent les feux de joie des significations multiples". 


\section{Bibliographie}

APOLlINAIRE, Guillaume. 1993. CEuvres en prose complètes I. Paris, NRF Gallimard "La Pléiade". APOLLINAIRE, Guillaume.1994. CEuvres en prose complètes II, Paris, NRF Gallimard "La Pléiade". APOLLINAIRE, Guillaume. 2001. CEuvres poétiques. Paris, NRF Gallimard "La Pléiade".

BARTHES, Roland. 1980. "Théorie du texte", Paris, Encyclopaedia Universalis, vol. XV, 1013-1017.

BAUdelaIRE. 1968. "De l'essence du rire", CEuvres Complètes, Paris, Seuil, "L'Intégrale".

BURGOS, Jean. 1982. Pour une poétique de l'imaginaire. Paris, Seuil.

BuRgos, Jean. 2009. Apollinaire et "L'Enchanteur pourrissant". Genèse d'une poétique. Clamart, Calliopées.

Deleuze, Gilles. (22 mars 1983), Les Cours de G. Deleuze à Vincennes-Saint-Denis: "Image mouvement-image temps" (date de consultation: le 15 mars 2010) <URL: http://www.webdeleuze.com/php/texte.php >.

SATO, Fumiro. 2004. "La réalité dans l'œuvre d'Apollinaire: autour des "Mamelles de Tirésias". Études de Langue et Littérature Françaises. Tokyo. № 84: p. 170-184.

TADIE, Jean-Yves. 1968. Le récit poétique. Paris, PUF "Écritures". 